\title{
Changes of the plasma metabolome of newly born piglets subjected to postnatal hypoxia and resuscitation with air
}

\author{
Rønnaug Solberg' ${ }^{1}$ Julia Kuligowski², Leonid Pankratov' ${ }^{1}$ Javier Escobar², Guillermo Quintás ${ }^{3}$, Isabel Lliso², \\ Ángel Sánchez-Illana ${ }^{2}$, Ola Didrik Saugstad' and Máximo Vento ${ }^{2,4}$
}

BACKGROUND: Perinatal hypoxic-ischemic brain damage is a major cause of mortality and morbidity in the neonatal period. Currently, limited ranges of biochemical tests assessing the intensity and duration of hypoxia are ready for clinical use. However, the need to initiate hypothermia therapy early after the clinical suspicion of hypoxic-ischemic encephalopathy requires the availability of early and reliable hypoxia markers. We have sought these biomarkers in an experimental model of hypoxia reoxygenation.

METHODS: Hypoxia and hypotension were induced in newborn piglets following a standardized model and reoxygenation was carried out using room air (RA). An untargeted liquid chromatography - time of flight mass spectrometry (LC-TOFMS) approach was used to assess changes in the metabolomic profile of plasma samples after intense hypoxia and upon reoxygenation.

RESULTS: At the end of hypoxia, the plasma metabolome showed an increased plasma concentration of analytes reflecting a metabolic adaptation to prolonged anaerobiosis. However, after resuscitation, metabolite levels returned to the starting values.

CONCLUSION: Severe hypoxia induces early, significant, and transient changes of specific metabolites in the plasma metabolome, which represent a snapshot of the biochemical adaptation of mammals to intense hypoxia. These metabolites could have applicability in predicting the severity of hypoxia in the clinical setting.

$\mathbf{P}$ erinatal hypoxia-ischemia (HI) is an injurious event that may precipitate a cascade of biochemical processes, which can lead to multiorgan failure including neuronal cell death evolving for hours, days, or even months (1). At present, clinical assessment which includes Apgar score, serum blood gases, and lactate analysis lack desired reliability both in the assessment of a good postnatal adaptation and to predict long-term neurocognitive outcome $(2,3)$. Both, amplitude-integrated electroencephalography and magnetic resonance imaging offer valuable information that can be predictive of outcome; however, they do not have the sensitivity needed to inform treatment. Moreover, in many centers, there is no easy access to these technologies (4). Therapeutic hypothermia has significantly improved prognosis of a substantial number of asphyxiated babies evolving to hypoxic-ischemic encephalopathy. However, the need to promptly initiate therapeutic hypothermia after the hypoxic-ischemic $(<6 \mathrm{~h})$ insult urges the need for diagnostic tools, which rapidly and reliably assess the degree of hypoxia thus helping to stratify patients and adopt clinical decisions (5). Biochemical evaluation of the severity of birth asphyxia has been traditionally performed using blood gases and lactate concentrations in cord blood at birth. However, in a study by Solberg et al. (6), changes in metabolites during neonatal hypoxia were analyzed and correlated with the duration of hypoxia using targeted metabolomics. Although blood lactate, $\mathrm{pH}$, and base excess increased in the first minutes after hypoxia, there was no significant correlation between these parameters and the duration of hypoxia. In contrast, ratios of alanine/branched chain amino acids and glycine/branched chain amino acids could significantly predict the duration and intensity of hypoxia. By combining these ratios with other metabolites such as intermediates of the Krebs cycle (succinate) and propionyl-L-carnitine (C3), the correlation of the duration of hypoxia was significantly increased (6).

In the present study, we have employed untargeted metabolomics for the analysis of metabolic changes. Untargeted metabolic profiling is defined as the holistic analysis of endogenous metabolites, providing metabolic information for phenotyping, or the understanding of physiological or pathophysiological mechanisms, and is commonly considered as a hypothesis free survey, which is frequently employed for the discovery of biomarkers (7). Several untargeted metabolomic studies have been carried out based on nuclear magnetic resonance spectra from urine samples from piglets subjected to hypoxia and reoxygenation. Skappak et al. (8) and Murgia et al. (9) found sets of metabolites capable of differentiating between normoxic and hypoxic groups. Many of these metabolites were involved

\footnotetext{
'Department of Pediatric Research, Institute for Surgical Research, University of Oslo, Oslo University Hospital - Rikshospitalet, Oslo, Norway; ${ }^{2}$ Neonatal Research Group, Health Research Institute Hospital La Fe, Valencia, Spain; ${ }^{3}$ Leitat Technological Center, Safety and Sustainability Division, Valencia, Spain; ${ }^{4}$ Division of Neonatology, University \& Polytechnic Hospital La Fe, Valencia, Spain. Correspondence: Máximo Vento (maximo.vento@uv.es) 
in the cellular energy metabolism or resulted from the effect of hypoxia on inflammation and deregulation of amino acid metabolism. Moreover, a recent study investigated metabolic changes in piglets' retina after an intense period of hypoxia (10). Untargeted liquid chromatography-time of flight mass spectrometry (LC-TOFMS) revealed that there was a significant increase in cytidine di-phosphate-choline, a precursor of phosphocholine in the retina after hypoxia (11). Although a promising candidate, no studies in easily available matrices such as plasma or urine have been yet performed.

The aim of this study was to identify early plasma biomarkers of hypoxia in a well-known piglet model (12) using an untargeted LC-TOFMS approach. Results could find potential application in the clinical setting for diagnosing, monitoring, and predicting the consequences of birth asphyxia and response to resuscitation and neuroprotective interventions.

\section{RESULTS}

\section{Cohort Characterization During the Experiment}

Table 1 summarizes the physiological parameters monitored during the experiment. No differences between control and experimental animals (RA group) were found for the basic biologic characteristics or for the clinical parameters at the initiation of the experiment ("Start hypoxia"). However, after 53.4( \pm 17) min of intense hypoxia $\left(\mathrm{FiO}_{2} 8 \%\right)$, as shown in Table 1 the experimental group had a significantly lower $\mathrm{pH}$, base excess, and blood pressure while no differences in heart rate were evidenced ("End hypoxia"). In the experimental group, however, resuscitation with RA rapidly improved clinical parameters at the end of the resuscitation procedure ("End resuscitation") except for an increased heart rate.

\section{Comparison of the Effect of Hypoxia Upon Plasma Samples}

Partial least squares discriminant analysis (PLSDA) models for assessing the effect of hypoxia and reoxygenation on the plasma metabolome were developed. A subset of 17 randomly selected samples of classes "Start hypoxia" and "End hypoxia" were used for the development of a PLSDA model to analyze the effect of hypoxia on the plasma metabolome, as described in the Methods section. The results from a permutation test confirmed the statistical significance of the multivariate discriminant model between groups using three latent variables (Wilcoxon test, $P$ value $<0.05$ ). This model was then used to identify which descriptors explained most of the observed difference. The elimination on noninformative variables from the metabolomic PLSDA model reduces its complexity, facilitates data visualization and understanding and, at the same time, improves the prediction performance of the PLSDA model. Using the variable influence in projection (13) to quantify the significance of each variable in the model, a subset of 46 differentiating variables was retained in the final model. Details on the variable selection process can be found in the Supplementary Material online. Table 2 summarizes $\mathrm{m} / \mathrm{z}$, $\mathrm{RTs}$, and the putative identifications of the differentiating variables between both groups using open databases, as well as the outcome ( $P$ value) from paired $t$-tests for the null hypothesis
Table 1. Physiological background data

\begin{tabular}{|c|c|c|}
\hline Parameter & Control & Room air \\
\hline Weight (g) & $1,810( \pm 173)$ & $1,889( \pm 127)$ \\
\hline Age $(h)$ & $28.7( \pm 3)$ & $25.6( \pm 4)$ \\
\hline Gender (M/F) & $3 / 3$ & $12 / 14$ \\
\hline Hypoxia (min) & 0 & $53.4( \pm 17)$ \\
\hline \multicolumn{3}{|l|}{$\mathrm{Hb} \mathrm{g} / 100 \mathrm{ml}$} \\
\hline Start hypoxia & $7.2( \pm 1.0)$ & $7.3( \pm 1.1)$ \\
\hline End of study & $6.7( \pm 0.8)$ & $6.8( \pm 0.05)$ \\
\hline \multicolumn{3}{|l|}{$\mathrm{pH}$} \\
\hline Start hypoxia & $7.41( \pm 0.04)$ & $7.44( \pm 0.07)(\mathrm{NS})$ \\
\hline End hypoxia & $7.42( \pm 0.03)$ & $6.86( \pm 0.07)(<0.001)$ \\
\hline End resuscitation & $7.44( \pm 0.03)$ & $7.16( \pm 0.07)(<0.001)$ \\
\hline $2 \mathrm{~h}>$ resuscitation & $7.46( \pm 0.03)$ & $7.39( \pm 0.08)(\mathrm{NS})$ \\
\hline $5 \mathrm{~h}>$ resuscitation & $7.42( \pm 0.05)$ & $7.40( \pm 0.09)(\mathrm{NS})$ \\
\hline $9 \mathrm{~h}>$ resuscitation & $7.44( \pm 0.08)$ & $7.40( \pm 0.06)(\mathrm{NS})$ \\
\hline \multicolumn{3}{|l|}{$\mathrm{BE}(\mathrm{mmol} / \mathrm{l})$} \\
\hline Start hypoxia & $2.25( \pm 2.8)$ & $1.89( \pm 3.4)(\mathrm{NS})$ \\
\hline End hypoxia & $1.98( \pm 2.7)$ & $-19.3( \pm 2.2)(<0.001)$ \\
\hline End resuscitation & $1.98( \pm 2.7)$ & $-14.8( \pm 2.4)(<0.001)$ \\
\hline $2 \mathrm{~h}>$ resuscitation & $2.33( \pm 2.6)$ & $-0.4( \pm 4.2)(0.05)$ \\
\hline $5 \mathrm{~h}>$ resuscitation & $0.66( \pm 4.2)$ & $-0.7( \pm 4.8)(\mathrm{NS})$ \\
\hline $9 \mathrm{~h}>$ resuscitation & $-0.01( \pm 4.7)$ & $-0.3( \pm 5.2)(\mathrm{NS})$ \\
\hline \multicolumn{3}{|c|}{ Mean arterial blood pressure (mmHg) } \\
\hline Start hypoxia & $49.4( \pm 4.9)$ & $54.4( \pm 8.1)(N S)$ \\
\hline End hypoxia & $48.2( \pm 7.3)$ & $22.7( \pm 8.1)(<0.001)$ \\
\hline End resuscitation & $48.2( \pm 7.3)$ & $41.9( \pm 11.0)(<0.01)$ \\
\hline $2 \mathrm{~h}>$ resuscitation & $46.7( \pm 6.2)$ & $47.5( \pm 11.3)(\mathrm{NS})$ \\
\hline $5 \mathrm{~h}>$ resuscitation & $47.6( \pm 8.3)$ & $44.2( \pm 9.3)(\mathrm{NS})$ \\
\hline $9 \mathrm{~h}>$ resuscitation & $47.0( \pm 13)$ & $45.7( \pm 10.8)(N S)$ \\
\hline \multicolumn{3}{|l|}{ Heart rate } \\
\hline Start hypoxia & $147( \pm 11)$ & $144( \pm 26)(\mathrm{NS})$ \\
\hline End hypoxia & $156( \pm 20)$ & $160( \pm 47)(\mathrm{NS})$ \\
\hline End resuscitation & $156( \pm 20)$ & $200( \pm 35)(<0.01)$ \\
\hline \multicolumn{3}{|l|}{$\mathrm{pO}_{2} \mathrm{kPa}$} \\
\hline Start hypoxia & $9.9(1.0)$ & $10.6(1.7)(\mathrm{NS})$ \\
\hline End hypoxia & $10.3(0.8)$ & $5.0(0.6)(<0.001)$ \\
\hline End resuscitation & $10.3(0.8)$ & $10.7(1.4)(\mathrm{NS})$ \\
\hline \multicolumn{3}{|l|}{$\mathrm{pCO} 2 \mathrm{kPa}$} \\
\hline Start hypoxia & $5.4(0.5)$ & $5.2(0.8)(\mathrm{NS})$ \\
\hline End hypoxia & $5.1(0.3)$ & $9.3(1.1)(<0.001)$ \\
\hline End resuscitation & $5.1(0.3)$ & $4.7(0.8)(\mathrm{NS})$ \\
\hline
\end{tabular}

Characterization of the study cohort before starting the experiment (Start hpoxia), at the end of the hypoxic period (End hypoxia), and at the end of resuscitation (End resuscitation). The corresponding time points for the control group not submitted to hypoxia are also represented (Control).Values are presented as mean $( \pm$ SD). $\mathrm{Hb}$, hemoglobin; comparison by Student's " $\mathrm{t}$ "test. NS, no significant. 


\section{Articles | Solberget al.}

Table 2. List of selected features differentiating plasma before and at the end of hypoxia sorted according to their variable influence in projection score value in the PLSDA model of "Start hypoxia"vs. "End hypoxia"

\begin{tabular}{|c|c|c|c|c|c|c|c|}
\hline $\mathrm{m} / \mathrm{z}$ & $\begin{array}{l}\mathrm{RT} \\
(\mathrm{min})\end{array}$ & $($ End $) /(\text { start })^{a}$ & $\begin{array}{l}\text { Molecular } \\
\text { formula }^{\mathrm{b}}\end{array}$ & $E(m / z)^{c}$ & Ion & Putative metabolite & Class \\
\hline 205.0280 & 2.71 & $6.2(P<0.05)$ & UC 7 & -- & -- & -- & -- \\
\hline 315.0171 & 2.71 & $5.07(P<0.05)$ & UC 7 & -- & -- & -- & -- \\
\hline 137.0453 & 2.84 & $4.98(P<0.05)$ & $\mathrm{C} 5 \mathrm{H} 5 \mathrm{~N} 4 \mathrm{O}+$ & 0.1 & {$[\mathrm{M}+\mathrm{H}]+$} & Hypoxanthine & Imidazopyrimidines \\
\hline 233.9801 & 2.70 & $3.35(P<0.05)$ & UC 7 & -- & -- & -- & -- \\
\hline 138.0474 & 2.84 & $2.92(P<0.05)$ & $\mathrm{C} 5 \mathrm{H} 5 \mathrm{~N} 4 \mathrm{O}+$ & -3.1 & {$[\mathrm{M}+\mathrm{H}]+$} & Hypoxanthine & Imidazopyrimidines \\
\hline 110.0344 & 2.84 & $3.03(P<0.05)$ & $\mathrm{C} 4 \mathrm{H} 4 \mathrm{~N} 3 \mathrm{O}+$ & -0.8 & {$[\mathrm{M}+\mathrm{H}-\mathrm{CHNO}]+$} & 6,8-Dihydroxypurine & Imidazopyrimidines \\
\hline 152.0558 & 2.28 & $4.1(P<0.05)$ & $\mathrm{C} 5 \mathrm{H} 6 \mathrm{~N} 5 \mathrm{O}+$ & -2.5 & {$[\mathrm{M}+\mathrm{H}]+$} & 8-Hydroxyadenine & Imidazopyrimidines \\
\hline 269.0858 & 2.86 & $4.96(P<0.05)$ & $\mathrm{C} 10 \mathrm{H} 13 \mathrm{~N} 4 \mathrm{O} 5+$ & -5.0 & {$[\mathrm{M}+\mathrm{H}]+$} & Inosine & Purine nucleosides \\
\hline 234.9872 & 2.72 & $1.98(P<0.05)$ & UC 7 & -- & -- & -- & -- \\
\hline 112.0492 & 2.09 & $2.4(P<0.05)$ & $\mathrm{C} 4 \mathrm{H} 6 \mathrm{~N} 3 \mathrm{O}+$ & -3.0 & {$[\mathrm{M}+\mathrm{H}-\mathrm{C} 5 \mathrm{H} 8 \mathrm{O} 4]+$} & Cytidine & Pyrimidine nucleosides \\
\hline 246.1686 & 5.84 & $2.4(P<0.05)$ & $\mathrm{C} 12 \mathrm{H} 24 \mathrm{NO} 4+$ & -3.2 & {$[\mathrm{M}+\mathrm{H}]+$} & Valerylcarnitine & Fatty acyls \\
\hline 582.2411 & 9.87 & $0.44(P<0.05)$ & UC 11 & -- & -- & -- & -- \\
\hline 162.1116 & 1.96 & $0.3(P<0.05)$ & $\mathrm{C} 7 \mathrm{H} 16 \mathrm{NO} 3+$ & -3.5 & {$[\mathrm{M}+\mathrm{H}]+$} & L-Carnitine & Fatty acyls \\
\hline 132.1011 & 3.43 & $2.22(P<0.05)$ & $\mathrm{C} 5 \mathrm{H} 12 \mathrm{~N}+$ & 5.5 & {$[\mathrm{M}+\mathrm{H}]+$} & Leucine & Carboxylic acids and derivatives \\
\hline 643.1492 & 9.80 & $0.55(P<0.05)$ & UC 10 & -- & -- & -- & -- \\
\hline 258.1086 & 1.93 & $0.6(P<0.05)$ & $\mathrm{C} 10 \mathrm{H} 16 \mathrm{~N} 3 \mathrm{O} 5+$ & 2.1 & {$[\mathrm{M}+\mathrm{H}]+$} & 5-Methyl-Cytidine & Pyrimidine nucleosides \\
\hline 727.8188 & 1.80 & $0.8(P<0.05)$ & UC 1 & -- & -- & -- & -- \\
\hline 607.2484 & 9.75 & $0.43(P<0.05)$ & KC28H44N2O8S+ & 2.6 & {$[\mathrm{M}+\mathrm{K}]+$} & Leukotriene F4 & Fatty acyls \\
\hline 119.0272 & 2.64 & $0.93(P<0.05)$ & UC 6 & -- & -- & -- & -- \\
\hline 426.3562 & 9.42 & $6.24(P<0.05)$ & $\mathrm{C} 25 \mathrm{H} 48 \mathrm{NO} 4+$ & -0.4 & {$[\mathrm{M}+\mathrm{H}]+$} & 11Z-Octadecenylcarnitine & Fatty acyls \\
\hline 584.2592 & 9.76 & $0.27(P<0.05)$ & UC 9 & -- & -- & -- & -- \\
\hline 466.3128 & 6.76 & $5.68(P<0.05)$ & $\mathrm{C} 26 \mathrm{H} 44 \mathrm{NO} 6+$ & -2.8 & {$[\mathrm{M}+\mathrm{H}]+$} & Glycocholic acid & Steroids and steroid derivatives \\
\hline 86.0964 & 3.18 & $1.83(P<0.05)$ & $\mathrm{C} 5 \mathrm{H} 12 \mathrm{~N}+$ & 5.5 & $\begin{array}{c}{[\mathrm{M}+\mathrm{H}-\mathrm{CH} 2 \mathrm{O} 2]+/} \\
{[\mathrm{M}]+}\end{array}$ & Leucine, neurine & $\begin{array}{c}\text { Carboxylic acids and derivatives/ } \\
\text { alkaloids and derivatives }\end{array}$ \\
\hline 726.9826 & 2.05 & $0.75(P<0.05)$ & UC 2 & -- & -- & -- & -- \\
\hline 712.9887 & 2.05 & $0.81(P<0.05)$ & UC 5 & -- & -- & -- & -- \\
\hline 141.0651 & 2.12 & $0.66(P<0.05)$ & $\mathrm{C} 6 \mathrm{H} 9 \mathrm{~N} 2 \mathrm{O} 2+$ & -2.5 & {$[\mathrm{M}+\mathrm{H}]+$} & 1,3-Dimethyluracil & Diazines \\
\hline 191.0389 & 1.89 & $1.6(P<0.05)$ & $\mathrm{Na} 2 \mathrm{C} 5 \mathrm{H} 9 \mathrm{~N} 2 \mathrm{O} 3+$ & -3.2 & {$[\mathrm{M}+2 \mathrm{Na}-\mathrm{H}]+$} & L-Glutamine & Carboxylic acids and derivatives \\
\hline 169.0343 & 2.31 & $1.91(P<0.05)$ & $\mathrm{C} 5 \mathrm{H} 5 \mathrm{~N} 4 \mathrm{O} 3+$ & -3.1 & {$[\mathrm{M}+\mathrm{H}]+$} & Uric acid & Alkaloids and derivatives \\
\hline 370.2930 & 7.79 & $1.65(P<0.05)$ & $\mathrm{C} 21 \mathrm{H} 40 \mathrm{NO} 4+$ & 0.3 & {$[\mathrm{M}+\mathrm{H}]+$} & cis-5-Tetradecenoylcarnitine & Fatty acyls \\
\hline 132.1012 & 3.19 & $1.54(P<0.05)$ & $\mathrm{C} 6 \mathrm{H} 14 \mathrm{NO} 2+$ & -4.6 & {$[\mathrm{M}+\mathrm{H}]+$} & Leucine & Carboxylic acids and derivatives \\
\hline 130.0491 & 1.90 & $1.6(P<0.05)$ & $\mathrm{C} 5 \mathrm{H} 8 \mathrm{NO} 3+$ & -1.3 & {$[\mathrm{M}+\mathrm{H}-\mathrm{NH} 3]+$} & L-Glutamine & Carboxylic acids and derivatives \\
\hline 147.0753 & 1.90 & $1.5(P<0.05)$ & $\mathrm{C} 5 \mathrm{H} 11 \mathrm{~N} 2 \mathrm{O} 3+$ & -4.2 & {$[\mathrm{M}+\mathrm{H}]+$} & L-Glutamine & Carboxylic acids and derivatives \\
\hline 788.9833 & 2.05 & $0.88(P<0.1)$ & UC 3 & -- & -- & -- & -- \\
\hline 527.0810 & 2.78 & $0.93(P=0.07)$ & UC 8 & -- & -- & -- & -- \\
\hline
\end{tabular}

"Mean paired ratio of concentrations at time points"Start hypoxia" and "End hypoxia" using data included in the PLSDA calibration set. 'bU stands for unidentified compound. " $\mathrm{m} / \mathrm{z}$ error (ppm) relative to the molecular ion in column "Molecular Formula".

,-- corresponding to a metabolite without biological significance; PLSDA, partial least squares discriminant analysis. 
that the paired mean differences between the metabolite levels at "Start hypoxia" and "End hypoxia" in the calibration set come from normal distributions with means equal to zero and unknown variances. Results indicated that the null hypothesis could be rejected at the default $5 \%$ significance level for a total of 44 variables.

The predictive performance of the discriminant model between "Start hypoxia" and "End hypoxia" based on the 46 selected variables was assessed using an external validation data set that included: (i) plasma obtained after reoxygenation of the piglets included in the calibration set; (ii) "Start hypoxia", "End hypoxia", and "Reox" (resuscitated) samples from nine piglets; and (iii) plasma samples from "Control" piglets. The validation set was in no way used for variable selection and model development and thus, it provided external figures of merit. Results of the predicted class values for both, calibration and validation sets are shown in Figure 1a. No differences for the control and the experimental group were found when comparing "Start hypoxia" and "Reox". On the other hand, the effect of hypoxia and reoxygenation on the plasma metabolome
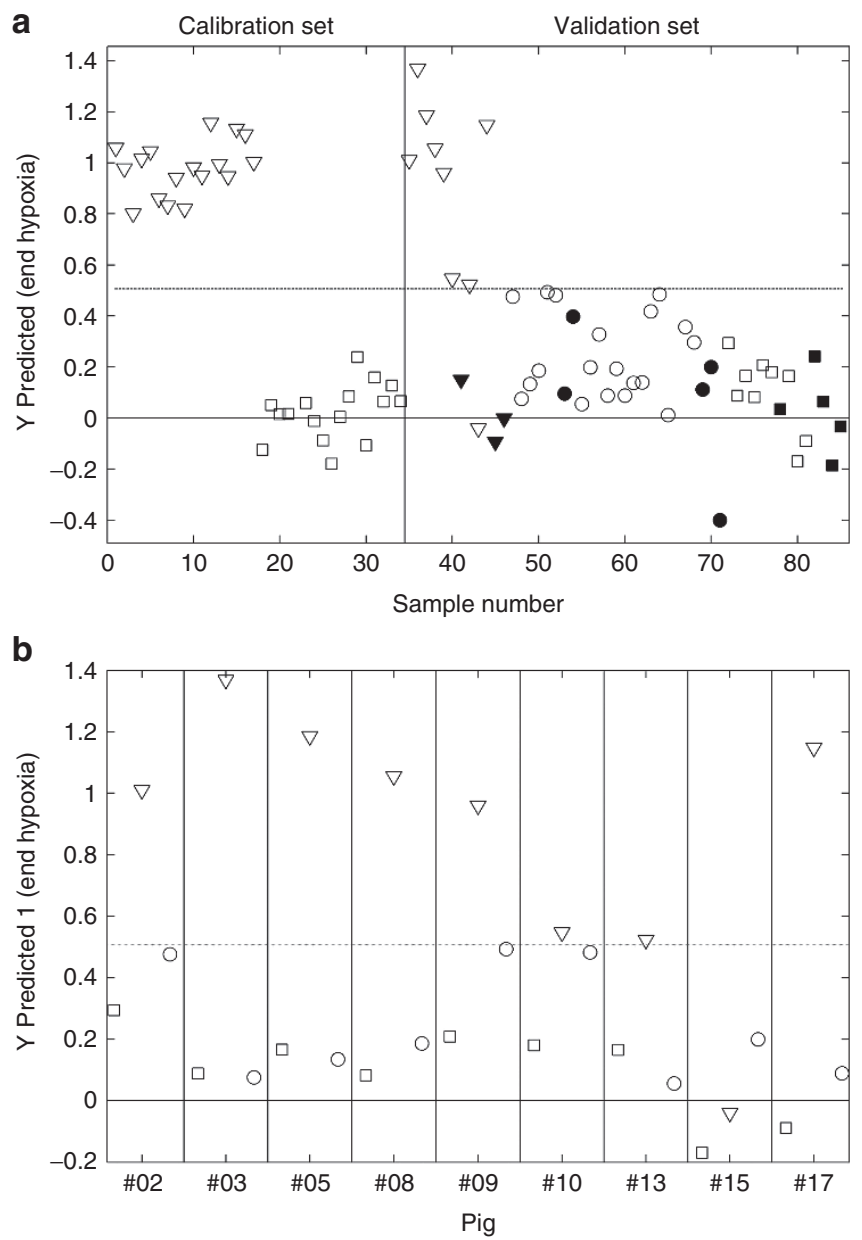

Figure 1. Predicted $y$-values for calibration and validation samples of groups "Start hypoxia" vs. "End hypoxia". (a) Calibration and full validation set. (b) Samples withdrawn from nine piglets at three different time-points. Note: black open triangles, End-RA; black, filled triangles, EndControl; black squares, Start-RA; black, filled squares, Start-Control; black open circles, Reox-RA; black, filled circles, Reox-Control. RA, room air. was clearly reflected in the predicted $y$ values for samples withdrawn from nine piglets at three different time-points. Results are shown in Figure 1b. Abnormal clinical observations during the experiment observed for piglet \#15 were reflected in the metabolomic profiles providing predicted $y$ values in good agreement with clinical records (see Figure 1b). Piglet \#15 had a long hypoxia time $(57 \mathrm{~min})$, suffered from severe metabolic acidosis ( $\mathrm{pH} 6.70$, base excess (BE) $-23 \mathrm{mmol} / \mathrm{l}^{2}$, lactate 19) and it recovered very slowly after hypoxia. In short, results obtained for the validation set confirmed the reliability of the metabolomic approach as it reflected the effect of the treatment and matched abnormal clinical observations.

Given the complexity of the results, we grouped identified metabolites in nine related clusters as indicated in Table 2. Boxplots shown in Figure 2 represent the most relevant changes for clusters of metabolites with a significance of at least $P<0.05$ between the values at "Start hypoxia" and "End hypoxia". Figure 2a represents carboxylic acids and derivatives including leucine, neurine, and L-glutamine; Figure $\mathbf{2 b}$ shows fatty acyls changes including carnitine, valeryl-carnitine, lynoleyl-carnitine, octadecenyl-carnitine, and proinflammatory metabolites such as leukotrienes; Figure $2 \mathrm{c}$ depicts changes in the levels of imidazopyrimidines, which predominantly represent hypoxanthine and adenine derivatives such as 8-hydroxyadenine, 6,8-dihydroxypurine, and guanine; Figure 2d shows pyrimidine nucleosides; Figure 2e represents alkaloids and derivatives; Figure $2 \mathrm{f}$ represents the cluster of diazines; Figure 2 $\mathrm{g}$ depicts quaternary ammonium salt levels, which are represented by choline; Figure $2 \mathrm{~h}$ shows purine nucleosides; and Figure 2i shows steroids and steroid derivatives. Metabolites classified according to the rate of fold increase of the concentration ratio at the "End hypoxia" vs. the "Start Hypoxia" are shown in Figure 3. Choline and derivatives (quaternary ammonium salts) rank in the first place with an 8.3-fold increase, followed by purine derivatives (7.9-fold increase), fatty acids (6.3-fold increase), glycocholic acid (5.6fold increase), and hypoxanthine (5.0-fold increase). After resuscitation, the plasma concentration of these metabolites returned to "Start hypoxia" values. This can have clinical implications in the ongoing search for biomarkers for hypoxia. At birth of an asphyxiated newborn, umbilical cord blood may be available for analysis and early risk stratification can be made toward intervention therapies.

Using autoscaled intensities of the 21 selected metabolites in the 17 piglets used for model development, a quantitative enrichment analysis (QEA) (14) was performed to obtain a first indication of which metabolic pathways might reflect the effect of the hypoxic insult in the plasmatic metabolic fingerprinting. Here, the pathway-associated metabolite sets library containing 88 metabolite sets based on normal metabolic pathways was selected (15). Results from the QEA provided a list of metabolite sets ordered according to their fold enrichmentcalculated as the ratio of actual Q-value/expected Q-value, and colored according to their $P$ values adjusted for multiple testing using Bonferroni and false discovery rate (see Figure 4). 
a

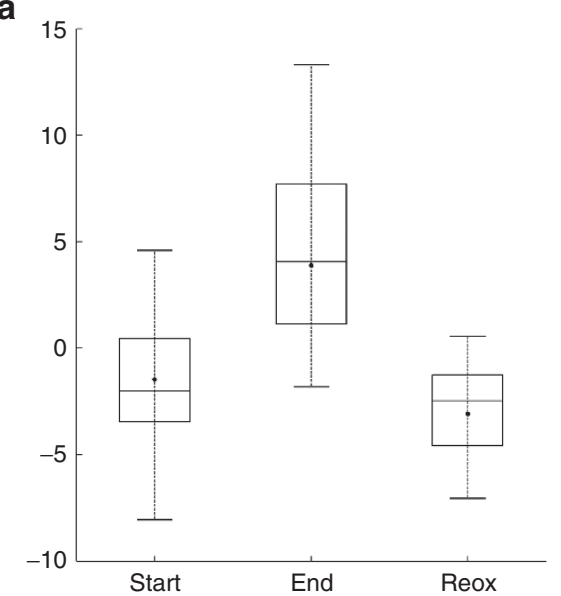

b
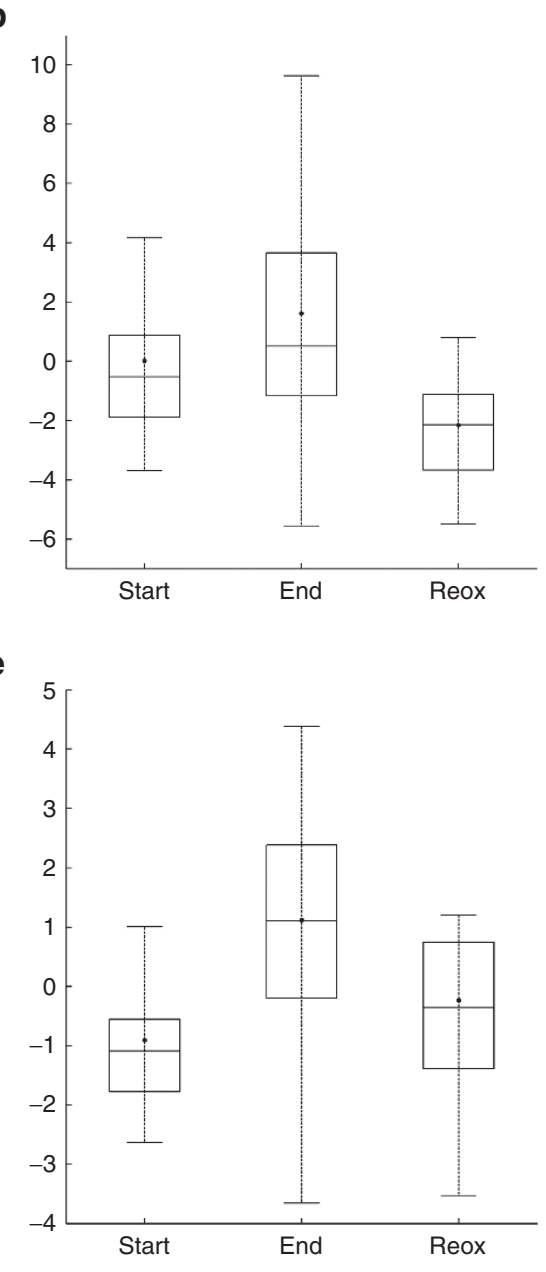

h

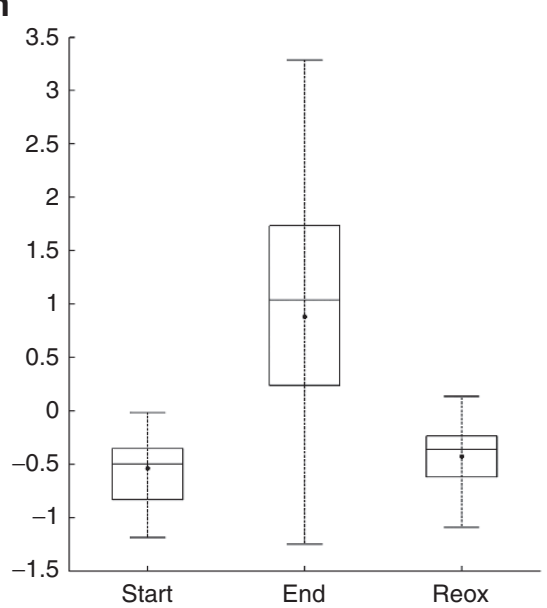

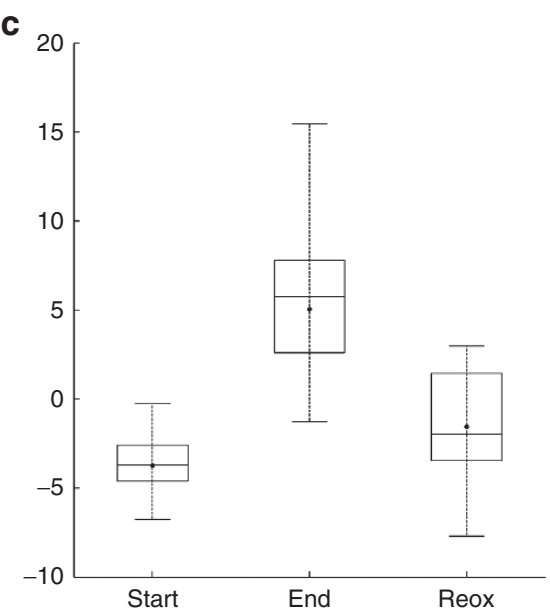

f

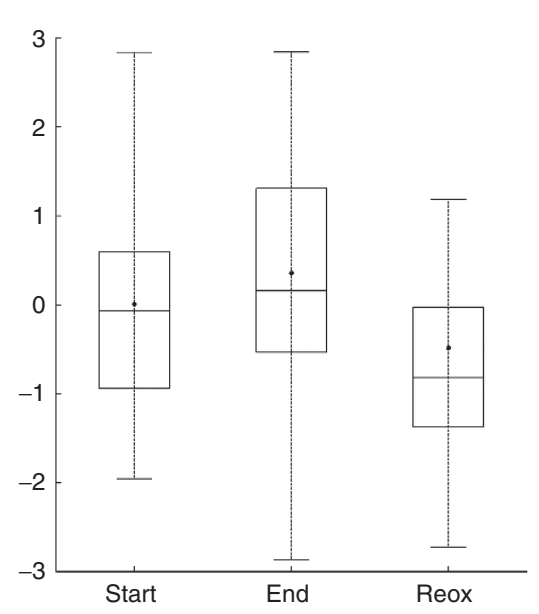

i

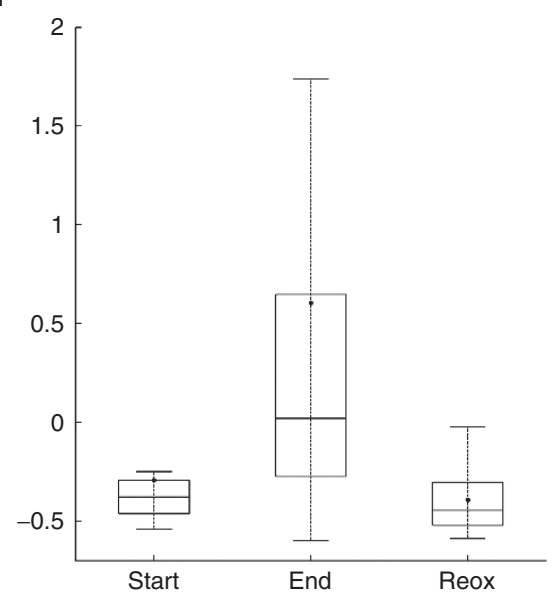

Figure 2. Boxplots represent the most relevant changes for clusters of metabolites with a significance of at least $P<0.05$ between the values at different time points ("Start hypoxia" and "End hypoxia") from the RA (room air) group. (a) Carboxylic acids and derivatives including leucine, neurine, and L-glutamine. (b) Fatty acyls changes including carnitine, valeryl-carnitine, lynoleyl-carnitine, octadecenyl-carnitine, and proinflammatory metabolites such as leukotrienes. (c) Changes in the levels of imidazopyrimidines which predominantly represent hypoxanthine and adenine derivatives such as 8-hydroxyadenine, 6,8-dihydroxypurine, and guanine. (d) Pyrimidine nucleosides. (e) Alkaloids and derivatives. (f) The cluster of diazines. (g) Quaternary ammonium salt levels which are represented by choline. (h) Purine nucleosides and (i) steroids and steroid derivatives. Note: Data were autoscaled; Start stands for "Start hypoxia"; End stands for "End hypoxia". 


\section{DISCUSSION}

Birth asphyxia is characterized by intermittent periods of ischemia and hypoxia that if prolonged enough can cause damage especially to oxyregulator tissues such as brain and myocardium (1). The success of moderate hypothermia is clearly related to timing of initiation of the therapy (less than $6 \mathrm{~h}$ after

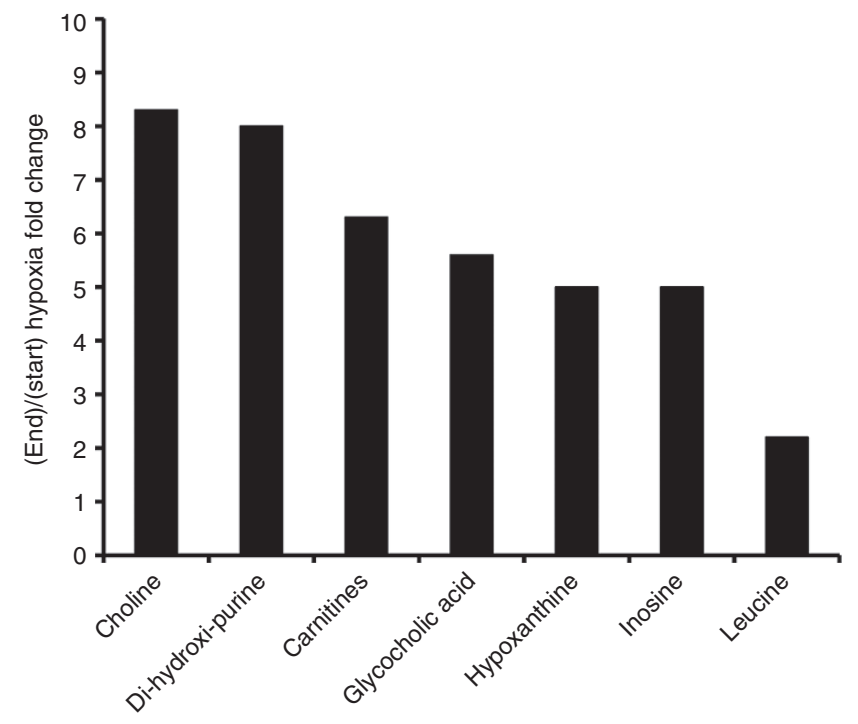

Figure 3. Results from the quantitative enrichment analysis using a subset of 21 differentiating metabolites reflecting the hypoxic insult in the plasma metabolomic profile. Note: NTC stands for number of total compounds in the metabolic pathway. birth) and severity of the insult $(4,5)$. While clinical evaluation in the delivery room as well as cord gases or lactate are easily available, they do not accurately reflect hypoxia in babies with moderate hypoxic-ischemic encephalopathy, which are the ones that mostly benefit from therapeutic hypothermia (1-5). The availability of reliable biomarkers could help clinicians to identify both time and severity of the hypoxic insult and establish a suitable therapy. We have detected significant metabolomic changes after exposure of newborn piglets to severe $\left(\mathrm{FiO}_{2}: 8 \%\right)$ and prolonged ( $\left.\sim 60 \mathrm{~min}\right)$ hypoxia. Remarkably, after completing resuscitation, there were no significant metabolomic changes in the intervention group reflecting transient metabolic changes. However, assessing the metabolomic profile during this immediate post-hypoxic-ischemic interval could be useful. Hence, if changes of hypoxia-ischemia persisted for some interval of time, it could be a signature that could facilitate identifying outborn infants at risk remote from delivery. Metabolomic changes due to prolonged ventilation and anesthesia were not found in the control group. Results obtained for the validation set confirmed the reliability of the metabolomic approach as it reflected the effect of the treatment and matched abnormal clinical observations.

\section{Biological Interpretation of the Metabolic Alterations Observed During Hypoxia}

The metabolic switch during hypoxia observed for the plasma sample composition followed by a recovery as shown in Figure 1, is simultaneous to the changes detected for

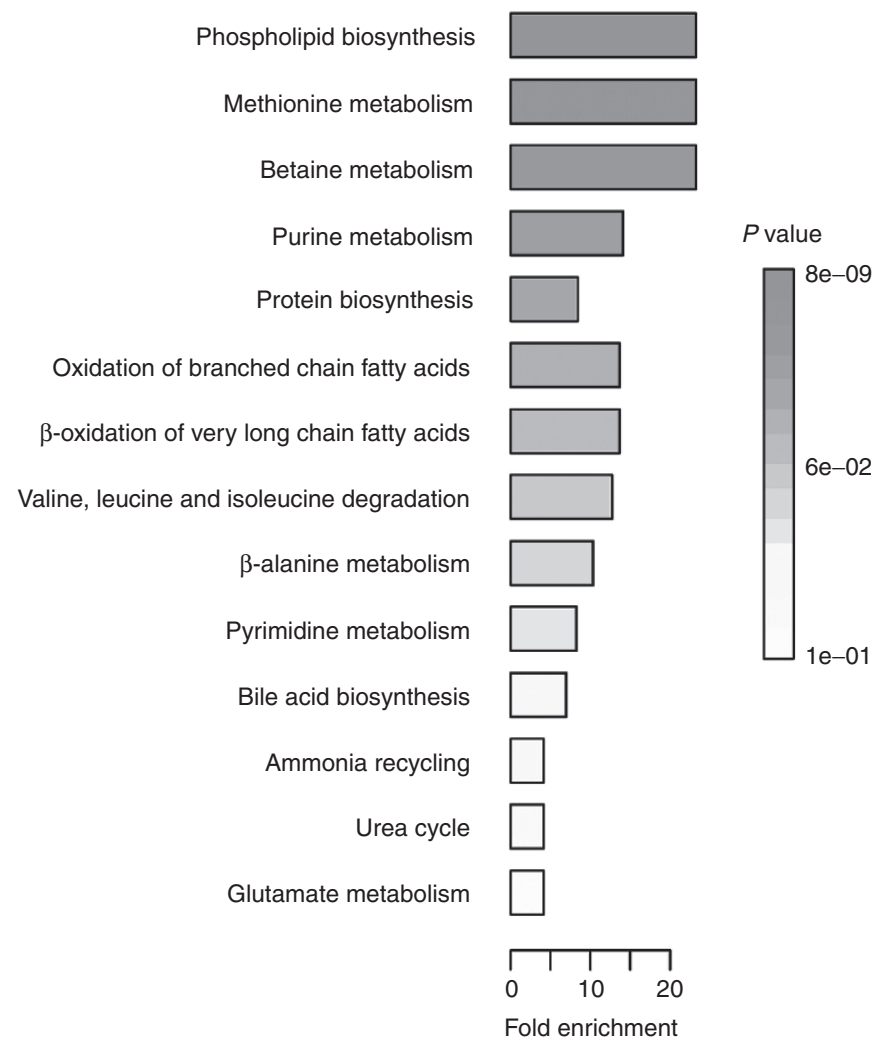

\begin{tabular}{|c|c|}
\hline NTC & Hits \\
\hline 19 & Choline \\
\hline 24 & Choline \\
\hline 10 & Choline \\
\hline 45 & Hypoxanthine, Inosine, Guanine, Uric acid, L-Glutamine \\
\hline 19 & L-Glutamine, L-Leucine \\
\hline 14 & L-Carnitine \\
\hline 14 & L-Carnitine \\
\hline 36 & L-Leucine \\
\hline 13 & Uracil \\
\hline 36 & Cytidine, Uracil, L-Glutamine \\
\hline 49 & Glycocholic acid \\
\hline 18 & L-Glutamine \\
\hline 20 & L-Glutamine \\
\hline 18 & L-Glutamine \\
\hline
\end{tabular}

$\begin{array}{lll}0 & 10 & 20\end{array}$

Figure 4. Concentration ratio (end hypoxia)/(start hypoxia) of the most significant analytes detected in hypoxic newborn piglets submitted to an $8 \%$ inspiratory fraction of oxygen for approximately $60 \mathrm{~min}$. 


\section{Articles | solberget al.}

physiological parameters currently employed in the clinical setting including lactate concentration, $\mathrm{BE}$, or mean arterial blood pressure of hypoxic animals. Nonetheless, LC-TOFMS results could unveil new insights into metabolic pathways that could play a major role in the adaptive response against acute hypoxia as well as novel and early biomarkers useful for clinical management and monitoring of birth asphyxia and resuscitation. Out of 46 differentiating variables, 28 variables could be identified putatively as metabolites $([\mathrm{M}+\mathrm{H}]+, 21)$, or their isotopes and adducts as shown in Table 2. In spite of the usefulness of this type of analysis, results could have been biased to the metabolic pathways containing metabolites covered by the selected metabolomic strategy (15). For many of the analyzed metabolites, there is a greater distribution at end hypoxia compared with the other time points. Due to genetic diversity, the piglets may react differently to severe and prolonged hypoxia. This is also shown in the distribution of the hypoxia time (53.4 $( \pm 17) \mathrm{min})$, the time needed to reach a decrease in mean arterial blood pressure $<20 \mathrm{mmHg}$ or a $\mathrm{BE}$ of $-20 \mathrm{mmol} / \mathrm{l}^{2}$.

Our results show that choline is the most significantly increased analyte during the hypoxic insult (Figures 3 and 4). Cytidine di-phosphate-choline is the limiting intermediate metabolite in the biosynthesis of phosphatidyl-choline and plays an important role in membrane formation and repair. However, from an energy metabolism point of view, under hypoxic conditions, the interaction between choline and diacyl-glycerol is reversed leading to the degradation of diacyl-glycerol to free fatty acids (16). Hence, metabolomics evidenced a substantial increase in free fatty acids also at the end of hypoxia. The increase of cytidine and uridine derivatives, free fatty acids, and choline in plasma supports the idea that the Kennedy pathway and the transport of such metabolites are significantly altered during intense and prolonged hypoxia and could be used as early biomarkers of relevant alterations in cell metabolism $(10,11)$. In addition, we found that levels of hypoxanthine, a hallmark of hypoxia $(17,18)$, as well as other intermediates of purine catabolism including 8-hydroxyadenine, inosine, 6,8-dihydroxypurine, guanine, and uric acid were increased in plasma of the hypoxic newborn piglets. These findings are in concordance with other publications pointing to the physiologic role of the purine catabolism in response to hypoxia $(17,18)$. In addition, this approach revealed the simultaneous increase of pyrimidine metabolism derivatives such as cytidine and uracil in plasma of hypoxic animals as well as the decrease of 5-methylcytidine and 1,3-dimethyluracil. Thus, it seems that not only purines, but also pyrimidine play an important role during acute hypoxia as well biological processes such as RNA methylation and/or de-methylation. An increase of the carnitine-fatty acids conjugates valeryl-carnitine, cis-5-tretradecenoylcarnitine, linoleyl-carnitine, and 11Z-octadecenyl-carnitine as well as a decrease of free carnitine in plasma of hypoxic animals immediately after the insult suggests a defensive metabolic response at mitochondrial level. In fact, in a hypoxic rat model, supplementation with L-carnitine significantly reduced brain lactate levels and lipid peroxidation byproducts thus indicating that carnitines may constitute defense mechanisms especially in oxyregulator tissues when confronted with a hypoxic insult (19). Furthermore, a rise of plasma branched chain amino acids during acute hypoxia could reflect an alteration of muscle or liver metabolism $(20,21)$. In addition, glycocholic acid, which is a biliary salt, was identified as a differentiating metabolite in this study, suggesting that liver function may be altered during acute hypoxia (22). Finally, an increase of the plasma content of glutamine after hypoxia was found. Glutamine is related to the glutamate metabolism, which is linked with aminotransferase reactions, ureagenesis, the tricarboxylic acid cycle, glutathione and $\gamma$-amino-butyric acid synthesis, and brain function $(23,24)$.

Seemingly, results of the present study are different from those previously described (6). However, there are substantial differences in both, the study design and the methodological approach to analyze blood samples obtained before and after hypoxia that could explain these differences. Yet, taking into account the metabolites that could be identified in the present study, the reported findings are still valuable for understanding the effect of hypoxia.

\section{Conclusions}

This untargeted LC-TOFMS metabolomic study of plasma samples from newborn piglets subjected to postnatal hypoxia has revealed a rapid and transient change of the plasmatic metabolic fingerprinting immediately after the hypoxic insult.

One hundred and twenty minutes after start of reoxygenation ("Reox"), plasma fingerprints were comparable to those observed before start of hypoxia. Out of 46 differentiating variables of a PLSDA model comparing plasma samples of groups "Start hypoxia" vs. "End hypoxia", 21 metabolites could be identified. Alterations of the purine catabolism, the pyrimidine metabolism, and the phospholipid synthesis (Kennedy pathway) have been observed. Other metabolites suffering changes are linked to mitochondrial function, $\beta$-oxidation, and neurotransmission.

Our results could have an application in different clinical conditions having as a common feature the presence of repetitive hypoxic events (apnea of prematurity), inflammatory processes (bronchopulmonary dysplasia, sepsis), or chronic hypoxic states (cyanotic congenital cardiac malformations). However, before the full potential of these candidate biomarkers is translated into the clinical setting for risk stratification or as early predictors for outcome, it needs further evaluation. For this purpose, we have launched a randomized controlled multicenter clinical trial in moderately to severely asphyxiated babies treated with whole body total moderate hypothermia, which includes metabolomic determinations immediately after birth (EUDRACT \#2011-005696-17). We aim to establish correlations between metabolomic profiles and electro-physiologic, magnetic resonance, and neurocognitive development (Bayley III, Wechsler Intelligence Scale for Children) scores.

\section{METHODS}

\section{Ethics Statement}

The animal study was carried out at Oslo University Hospital (Norway). The Norwegian Council for Animal Research approved the experimental protocol (approval number 3399). The animals were cared for and handled in accordance with the European Guidelines 


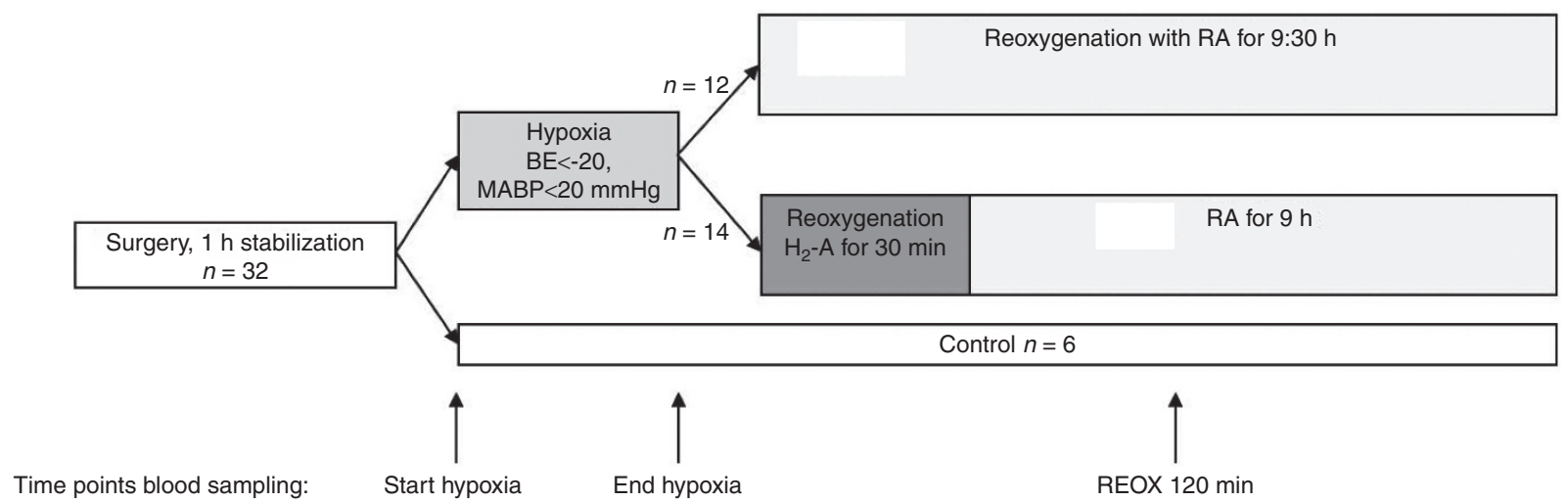

Figure 5. Scheme of the experimental protocol.

for Use of Experimental Animals by researchers certified by the Federation of European Laboratory Animals Science Association.

\section{Chemicals and Reagents}

All solvents were of LC-MS grade and were purchased from Scharlau (Barcelona, Spain). Additives and standards were purchased from Sigma-Aldrich Quimica SA (Madrid, Spain). Phenylalanine- $\mathrm{D}_{5}$ (Phe$\mathrm{D}_{5}$ ) and methionine- $\mathrm{D}_{3}$ (Meth- $\mathrm{D}_{3}$ ) supplied by Cambridge Isotope Laboratories (Tewksbury, MA) with purities $>98 \%$ were employed as internal standards .

\section{Animal Model}

\section{Surgical preparation and anesthesia.}

Thirty-two newborn Noroc (LyxLD) pigs were included in the study, the inclusion criteria being aged between 12 and $36 \mathrm{~h}$, hemoglobin $(\mathrm{Hb})$ levels $>5 \mathrm{~g} / \mathrm{dl}$, and good general conditions. Anesthesia was induced with Sevofluran 5\% before an ear vein was cannulated. Thereafter, Sevofluran was disconnected and the piglets were given Pentobarbital sodium $20 \mathrm{mg} / \mathrm{kg}$ and Fentanyl $50 \mathrm{mg} / \mathrm{kg}$ iv as bolus injections. Anesthesia was maintained by continuous infusion of fentanyl $(50 \mu \mathrm{g} / \mathrm{kg} / \mathrm{h})$ and midazolam $(0.25 \mathrm{mg} / \mathrm{kg} / \mathrm{h})$. The piglets were orally intubated, ventilated, and surgically prepared as described by Andresen et al. (12).

\section{Experimental Protocol}

After 60 min of stabilization, the piglets were randomized either to the hypoxia and reoxygenation group $(n=26)$ or the control group $(n=$ 6) going through the same procedures and observation times (anesthesia, surgery, ventilation, and sample collection) but no exposure to hypoxia.

Hypoxemia and subsequently hypoxia-ischemia was achieved by ventilation with a gas mixture of $8 \% \mathrm{O}_{2}$ in $\mathrm{N}_{2}$ until either the mean arterial blood pressure decreased to $<20 \mathrm{~mm}^{2} \mathrm{Hg}$ or the $\mathrm{BE}$ reached $-20 \mathrm{mmol} / \mathrm{l}^{2}$. To imitate perinatal asphyxia, $\mathrm{CO}_{2}$ was added during hypoxemia aiming at a $\mathrm{PaCO}_{2}$ of $8.0-9.5 \mathrm{kPa}(60-71.3 \mathrm{mmHg})$. After 30 min of reoxygenation, all animals were observed for $9 \mathrm{~h}$ receiving $\mathrm{RA}$ and kept normocapnic $\left(\mathrm{PaCO}_{2} 4.5-5.5 \mathrm{kPa}(33.8-41.3 \mathrm{mmHg})\right.$ ) with continuous surveillance of blood pressure, saturation, pulse, temperature, and blood gas measurements. The experimental study design is visualized in Figure 5.

Blood samples for metabolomic profiling were taken in ethylenediamine-tetraacetic acid Vacutainer blood collection tubes before start of hypoxia ("Start hypoxia"), at the end of hypoxia ("End hypoxia"), and $120 \mathrm{~min}$ after end of hypoxia ("Reox") (Figure 5) and at the corresponding time points for control piglets. Blood drawn for testing was replaced by saline $1.5 \times$ the volume drawn. Plasma was obtained immediately after sampling by centrifuging whole blood samples at $2,000 \mathrm{~g}$ for $10 \mathrm{~min}$ at $4{ }^{\circ} \mathrm{C}$ and stored at $-80{ }^{\circ} \mathrm{C}$ until analysis. At the end of the observation time, the animals were given an overdose of pentobarbital (150 mg/kg IV).

\section{LC-TOFMS Metabolic Profiling}

Plasma samples were thawed on ice. $150 \mu \mathrm{l}$ of cold $\left(4{ }^{\circ} \mathrm{C}\right)$ acetonitrile were added to $50 \mu \mathrm{l}$ of plasma, followed by homogenizing on a
Vortex mixer. Samples were centrifuged at a speed of $10,000 \mathrm{~g}$ at $4{ }^{\circ} \mathrm{C}$ during $10 \mathrm{~min}$. Twenty-five microliters of supernatant were added to $100 \mu \mathrm{l}$ of internal standards solution containing $5 \mu \mathrm{mol} / 1 \mathrm{Phe}-\mathrm{D}_{5}$ and $10 \mu \mathrm{mol} / \mathrm{l}$ Meth- $\mathrm{D}_{3}$ in $\mathrm{H}_{2} \mathrm{O}(0.1 \% \mathrm{v} / \mathrm{v}$ formic acid $)$. Hundred microliters of aliquots of the obtained sample extracts were transferred into $200 \mu \mathrm{l}$ capped glass vials and placed in the refrigerated auto-sampler compartment.

Metabolomic profiling of the plasma extracts was performed on a 1200 RRLC Series Agilent chromatograph (Palo Alto, CA) using a Zorbax SB-C8 $(3 \times 150 \mathrm{~mm}, 3.5 \mu \mathrm{m}$, Agilent $)$ column. Mass spectrometry detection was performed using a 5600-TripleTOF MS spectrometer (ABSciex, Framingham, MA) in the positive ionization mode $\left(\mathrm{ESI}^{+}\right)$.

Peak tables were generated using the XCMS software (The Scripps Research Institute, La Jolla, CA). Data analysis including principal component analysis and PLSDA was carried out in Matlab 2012b (The Mathworks, Natick, MA) using in-house written functions and the PLS Toolbox 7.0 from Eigenvector Research (Wenatchee, WA). QEA was performed using MetaboAnalyst 2.0 (McGill University, Montreal, Quebec) (25). QEA evaluates whether a set of functionally related metabolites are significantly enriched, similarly as it is done using Gene Set Enrichment Analysis (26,27), using a collection of metabolite set libraries and importance measures (as $\mathrm{Q}_{\text {stat }}(28)$ ) calculated for each compound.

Molecular formula were assigned employing the PeakView software (version 1.1.1.2) from AB Sciex (Framingham, MA) with a $\mathrm{m} / \mathrm{z}$ tolerance of $8 \mathrm{ppm}$ and an intensity tolerance of $\leq 10 \%$ taking into account the agreement with theoretical isotopic profiles. Metabolite putative identification based on MS data was performed using the human metabolome database (http://www.hmdb.ca/) with a spectral mass tolerance of $\pm 2.5 \mathrm{mDa}$.

Details on the LC-TOFMS metabolic profiling can be found in the Supplementary Figure S1 online and Supplementary Figure S2 online.

\section{Data Quality Assessment}

Instrumental effects in long LC-TOFMS sample batches might lead to increased variability and decreased power to detect biologically meaningful responses (29). Instrument performance was assessed based on: (i) relative SD (RSD\%) of internal standards intensity, mass accuracy, and retention times; (ii) relative SD in QC samples (QC-RSD\%), and (iii) the presence of sample trends in the principal component 1 vs. principal component 2 scores plot from a principal component analysis model calculated using the whole sample set.

Data quality was found adequate after the elimination of two outliers (i.e., \#28-Reox and \#18-Start) excluded from further analysis. Data quality parameters are discussed in the Supplementary Material online.

\section{Metabolomic Analysis of Plasma Samples}

Supervised PLSDA was used to assess the effect of hypoxia and reoxygenation on the plasma metabolome and to eventually identify a subset of discriminant metabolites. A PLSDA model was developed using a subset of samples to focus on differences among sample subgroups "Start hypoxia" and "End hypoxia". During PLSDA model development, 


\section{Articles | Solberget al.}

the optimal number of latent variables was selected from the mean average classification error obtained after 20 random fourfold cross validations (CVs). Then, the statistical significance of the PLSDA figures of merit was evaluated by permutation testing. Accordingly, cross validation errors calculated using the real class labels were compared to reference null distributions of cross validation values obtained using randomly permuted class labels (number of permutations $=500$ ).

\section{SUPPLEMENTARY MATERIAL}

Supplementary material is linked to the online version of the paper at http:// www.nature.com/pr

\section{ACKNOWLEDGMENTS}

We would like to thank the Servicio de Soporte a la Investigación Experimental (SCSIE) of the University of Valencia (Spain).

\section{STATEMENT OF FINANCIAL SUPPORT}

J.E. and J.K. acknowledge their personal grants (Sara Borrell CD11/00154, CD12/00667) from Instituto Carlos III (Spanish Ministry of Economy and Competitiveness). I.L. is grateful for the financial support from the Laerdal Foundation (Stavanger; Norway). M.V. acknowledges the FISPI14/0433 grant from Instituto Carlos III and EC11-246 from the Spanish Ministry of Health, Social Services and Equality. This project was carried out with financial support from the Research Network on Maternal and Child Health and Development Retic Red SAMID RD12/0026/11 and FEDER funding from the European Union (Instituto Carlos III, Ministry of Economy and Competitiveness, Spain).

Disclosure: The authors declare that there are no conflicts of interest.

\section{REFERENCES}

1. Gunn AJ, Bennet L. Fetal hypoxia insults and patterns of brain injury: insights from animal models. Clin Perinatol 2009;36:579-93.

2. Grivell RM, Alfirevic Z, Gyte GM, Devane D. Antenatal cardiotocography for fetal assessment. Cochrane Database Syst Rev 2012;12:CD007863.

3. Yeh P, Emary K, Impey L. The relationship between umbilical cord arterial $\mathrm{pH}$ and serious adverse neonatal outcome: analysis of 51,519 consecutive validated samples. BJOG 2012;119:824-31.

4. Shankaran S, Pappas A, McDonald SA, et al.; Eunice Kennedy Shriver NICHD Neonatal Research Network. Childhood outcomes after hypothermia for neonatal encephalopathy. N Engl J Med 2012;366:2085-92.

5. Committee on Fetus and Newborn, Papile L-A, Baley JE, et al. Hypothermia and neonatal encephalopathy. Pediatrics 2014;133:1146-50.

6. Solberg R, Enot D, Deigner HP, et al. Metabolomic analyses of plasma reveals new insights into asphyxia and resuscitation in pigs. PLoS One 2010;5:e9606.

7. Yin P, Xu G. Current state-of-the-art of nontargeted metabolomics based on liquid chromatography-mass spectrometry with special emphasis in clinical applications. J Chromatogr A 2014;1374:1-13.

8. Skappak C, Regush S, Cheung PY, Adamko DJ. Identifying hypoxia in a newborn piglet model using urinary NMR metabolomic profiling. PLoS One 2013;8:e65035.

9. Murgia F, Noto A, Iacovidou N, et al. Is the quickness of resuscitation after hypoxia influenced by the oxygen concentration? Metabolomics in piglets resuscitated with different oxygen concentrations. J Pediatr Neonatal Individ Med JPNIM 2013;2:e020233.

10. Solberg R, Escobar J, Arduini A, et al. Metabolomic analysis of the effect of postnatal hypoxia on the retina in a newly born Piglet model. PLoS One 2013;8:e66540.
11. Fiedorowicz M, Makarewicz D, Stańczak-Mrozek KI, Grieb P. CDP-choline (citicoline) attenuates brain damage in a rat model of birth asphyxia. Acta Neurobiol Exp (Wars) 2008;68:389-97.

12. Andresen JH, Carlsen B, Solberg R, et al. Newborn piglets exposed to hypoxia after nicotine or saline pretreatment: long-term effects on brain and heart. J Matern Fetal Neonatal Med 2009;22:161-8.

13. Gromski PS, Xu Y, Correa E, Ellis DI, Turner ML, Goodacre R. A comparative investigation of modern feature selection and classification approaches for the analysis of mass spectrometry data. Anal Chim Acta 2014;829:1-8.

14. Xia J, Wishart DS. MSEA: a web-based tool to identify biologically meaningful patterns in quantitative metabolomic data. Nucleic Acids Res 2010;38(Web Server issue):W71-7.

15. Lourenço AB, Roque FC, Teixeira MC, Ascenso JR, Sá-Correia I. Quantitative $1 \mathrm{H}-\mathrm{NMR}$-metabolomics reveals extensive metabolic reprogramming and the effect of the aquaglyceroporin FPS1 in ethanol-stressed yeast cells. PLoS One 2013;8:e55439.

16. Gibellini F, Smith TK. The Kennedy pathway-de novo synthesis of phosphatidylethanolamine and phosphatidylcholine. IUMBM Life 2012;2: 414-28.

17. Saugstad OD. Hypoxanthine as a measurement of hypoxia. Pediatr Res 1975;9:158-61.

18. Saugstad OD. Hypoxanthine as an indicator of hypoxia: its role in health and disease through free radical production. Pediatr Res 1988;23:143-50.

19. Rauchová H, Vokurková M, Koudelová J. Hypoxia-induced lipid peroxidation in the brain during postnatal ontogenesis. Physiol Res 2012;61 Suppl 1:S89-101.

20. Dehne N, Brüne B. Sensors, transmitters, and targets in mitochondrial oxygen shortage-a hypoxia-inducible factor relay story. Antioxid Redox Signal 2014;20:339-52.

21. Muratsubaki H, Yamaki A. Profile of plasma amino acid levels in rats exposed to acute hypoxic hypoxia. Indian J Clin Biochem 2011;26:416-9.

22. Chiang JY. Bile acid metabolism and signaling. Compr Physiol 2013;3:1191-212.

23. Kelly A, Stanley CA. Disorders of glutamate metabolism. Ment Retard Dev Disabil Res Rev 2001;7:287-95.

24. Newsholme P, Procopio J, Lima MM, Pithon-Curi TC, Curi R. Glutamine and glutamate-their central role in cell metabolism and function. Cell Biochem Funct 2003;21:1-9.

25. Xia J, Mandal R, Sinelnikov IV, Broadhurst D, Wishart DS. MetaboAnalyst 2.0-a comprehensive server for metabolomic data analysis. Nucleic Acids Res 2012;40(Web Server issue):W127-33.

26. Subramanian A, Tamayo P, Mootha VK, et al. Gene set enrichment analysis: a knowledge-based approach for interpreting genome-wide expression profiles. Proc Natl Acad Sci USA 2005;102:15545-50.

27. Nam D, Kim SY. Gene-set approach for expression pattern analysis. Brief Bioinform 2008;9:189-97.

28. Goeman JJ, van de Geer SA, de Kort F, van Houwelingen HC. A global test for groups of genes: testing association with a clinical outcome. Bioinformatics 2004;20:93-9.

29. Wang SY, Kuo CH, Tseng YJ. Batch Normalizer: a fast total abundance regression calibration method to simultaneously adjust batch and injection order effects in liquid chromatography/time-of-flight mass spectrometrybased metabolomics data and comparison with current calibration methods. Anal Chem 2013;85:1037-46. 